\title{
Photon-Statistics Excitation Spectroscopy of a Quantum-Dot Micropillar Laser
}

\author{
T. Kazimierczuk, ${ }^{15}$ J. Schmutzler, ${ }^{1}$ M. Aßmann, ${ }^{1, *}$ C. Schneider, ${ }^{2}$ M. Kamp, ${ }^{2}$ S. Höfling, ${ }^{2,3}$ and M. Bayer ${ }^{1,4}$ \\ ${ }^{1}$ Experimentelle Physik 2, Technische Universität Dortmund, 44221 Dortmund, Germany \\ ${ }^{2}$ Technische Physik, Physikalisches Institut, Wilhelm Conrad Röntgen Research Center for Complex Material Systems, \\ Universität Würzburg, D-97074 Würzburg, Germany \\ ${ }^{3}$ SUPA, School of Physics and Astronomy, University of St. Andrews, St. Andrews KY16 9SS, United Kingdom \\ ${ }^{4}$ A. F. Ioffe Physical-Technical Institute, Russian Academy of Sciences, St Petersburg 194021, Russia \\ ${ }^{5}$ Institute of Experimental Physics, Faculty of Physics, University of Warsaw, ul. Pasteura 5, 02-093 Warsaw, Poland
}

(Received 4 November 2014; published 7 July 2015)

\begin{abstract}
We introduce photon-statistics excitation spectroscopy and exemplarily apply it to a quantum-dot micropillar laser. Both the intensity and the photon number statistics of the emission from the micropillar show a strong dependence on the photon statistics of the light used for excitation of the sample. The results under coherent and pseudothermal excitation reveal that a description of the laser properties in terms of mean input photon numbers is not sufficient. It is demonstrated that the micropillar acts as a superthermal light source when operated close to its threshold. Possible applications for important spectroscopic techniques are discussed.
\end{abstract}

DOI: 10.1103/PhysRevLett.115.027401

PACS numbers: 78.67.Hc, 42.65.Sf, 78.47.D-, 78.55.Cr

Optical spectroscopy is one of the most important experimental tools when it comes to studies of the elemental processes in fields ranging from physics to biology. While usually the spectral and temporal properties of light fields are considered to be of highest interest, especially for studies of coherence properties, the quantum statistics of light fields matter as well [1-3]. During the last few years, experimental techniques aimed at studies of photon number statistics have improved significantly [4-11], as has the fundamental understanding of photon number statistics [12]. Recently, some emphasis has also been placed upon using the quantum statistics of the excitation light field as a degree of freedom in spectroscopy [13-16] and quantum-optical spectroscopy has recently been applied to reveal significant physical effects and new quasiparticles, such as dropletons $[17,18]$. The lowest-order quantum statistics beyond the mean value can be expressed via the second-order correlation function

$$
g^{(2)}(\tau)=\frac{\left\langle\hat{a}^{\dagger}(t) \hat{a}^{\dagger}(t+\tau) \hat{a}(t) \hat{a}(t+\tau)\right\rangle}{\langle\hat{n}(t)\rangle\langle\hat{n}(t+\tau)\rangle},
$$

where $\hat{a}^{\dagger}$ and $\hat{a}$ denote the photon creation and annihilation operators, $t$ represents the detection time of a photon, and $\tau$ gives the delay until the detection of a second photon. For different light fields of the same mean photon number, $g^{(2)}(0)$ is an indicator of the relative ratio of the variance of the photon number distribution to its mean, so a large $g^{(2)}(0)$ characterizes a noisy light field. For example, thermal, coherent, and single photon states are identified by $g^{(2)}(0)$ values of 2,1 , and 0 , respectively, which corresponds to photons arriving in bunches, independent of each other or well separated from each other, respectively. In principle, a description in terms of higher-order moments of the photon number distribution corresponding to a hierarchy of correlation functions up to arbitrary order can be used to completely characterize a light field, but in the following we will restrict ourselves to the second order as the most significant correlation.

As has been pointed out by some of the authors recently, systems showing a thresholdlike behavior in their response to coherent optical excitation should yield a clear signature of that nonlinearity already at significantly lower excitation densities when excited using thermal light [14]. Here we demonstrate this effect for a quantum-dot based verticalcavity surface-emitting laser. We find a significant increase in noise of almost 1 order of magnitude at around $20 \%$ of the threshold excitation density and show that this result agrees reasonably with our predictions by comparing the response to a thermal light field with the expected thermal response projected from the measured input-output curve for coherent excitation.

It is well known that the response of a system to excitation by a light field with arbitrary quantum statistics can be expressed in the density matrix formalism using the response to coherent states $\{|\alpha\rangle\}$ as a basis in phase space [19]. This is routinely done using the GlauberSudarshan distribution $P(\alpha)$ :

$$
\hat{\rho}=\int P(\alpha)|\alpha\rangle\langle\alpha| d^{2} \alpha .
$$

While $P(\alpha)$ may be nonanalytic or partially negative in the general case, it is well behaved for thermal light, in which we are interested. A single thermal mode yields a normal distribution in the basis of coherent states:

$$
P_{\mathrm{th}}(\{|\alpha\rangle\})=\frac{1}{\pi\langle n\rangle} e^{-\left(\alpha^{2} /\langle n\rangle\right)},
$$


which results in the well-known thermal photon number distribution:

$$
p_{\text {th }}(n)=\frac{\langle n\rangle^{n}}{(1+\langle n\rangle)^{n+1}} .
$$

In the limit of short pulses, where the coherence time is not shorter than the pulse duration, it is possible to interpret this distribution as a distribution of the photon numbers found in an ensemble of identically prepared thermal light pulses. We are operating well within this limit for the excitation light field. If the response of the system to excitation with a well-defined photon number was known, it would be straightforward to construct the response to thermal excitation by weighting the responses to Fock state excitation according to the weights given by Eq. (4). Of course, Fock states with photon numbers in our range of interest $\left(10^{6}-10^{7}\right.$ photons per pulse) are not experimentally accessible. However, measuring the full probability distribution histograms requires a huge number of measurements. For the large photon numbers considered here, coarse grained probability distributions are sufficient. Coarse grained probability distributions $P(n)$ can be obtained by binning $b$ adjacent values from the initial distribution together:

$$
P^{\prime}(n)=\sum_{k=n b}^{(n+1) b-1} p(k)
$$

Laser pulses show a Poissonian photon number distribution

$$
p(n)=\frac{\langle n\rangle^{n} e^{-\langle n\rangle}}{n !},
$$

with a standard deviation of $\sqrt{\langle n\rangle}$ around the mean value. For reasonable bin sizes of about $b=10^{4}$ the width of the distribution becomes smaller than the size of a bin and the difference between the binned probability distributions for coherent and Fock states of the same mean photon number becomes negligible. Accordingly, it is possible to construct the response to excitation with thermal light by using the weighted responses to a coherent light field of mean photon number $\langle n\rangle$ instead of using Fock states without introducing significant errors.

When constructing the thermal response from the response to coherent excitation, it is necessary to use the same binning size. For a thermal photon number distribution (4), it is still possible to calculate the binned sum of probabilities (5) exactly [20]:

$$
P_{\mathrm{th}}^{\prime}(n)=P^{\prime}(0) e^{-n b \ln (1+[1 /\langle n\rangle])} .
$$

In the case of large mean photon numbers, this expression can be simplified as follows:

$$
P_{\mathrm{th}}^{\prime}(n)=P^{\prime}(0) e^{-(n b /\langle n\rangle)} .
$$

The probability distributions derived in this section form the basis for the experimental procedure outlined in the next sections.

In principle, any nonlinear system is sensitive to photonstatistics excitation spectroscopy. We chose a microcavity laser as a proof-of-principle device because it allows us to work at low light levels. In that regime it is easier to create and maintain exact thermal photon number statistics as any influence of nonlinear effects created by the optics used are unlikely. The cylindrical micropillar laser studied here has a diameter of $8 \mu \mathrm{m}$ and was grown by molecular beam epitaxy and etched by electron-cyclotron-resonance reactive ion etching. The distributed Bragg reflector consists of 26 upper and 33 lower alternating layers of $\mathrm{AlAs}(74 \mathrm{~nm})$-GaAs $(68 \mathrm{~nm}) \lambda / 4$ pairs. The active medium consists of a single layer of self-assembled AlGaInAs quantum dots with a density of $\approx 6 \times 10^{9} \mathrm{~cm}^{-2}$. The sample was mounted on the cold finger of a helium-flow cryostat and kept at a temperature of $10 \mathrm{~K}$. For nonresonant optical excitation, we use a Martienssen lamp [21] consisting of laser light provided by a Ti-sapphire laser emitting pulses with a duration of $\approx 100 \mathrm{fs}$ at a repetition rate of $75.39 \mathrm{MHz}$ and a wavelength of $790 \mathrm{~nm}$ scattered by a rotating ground glass disk as our pseudothermal light source [22]. The laser light is focused onto the ground glass disk using a $50 \mathrm{~mm}$ focal length lens. The ground glass disk rotates at a frequency of approximately $8 \mathrm{~Hz}$. We collect a small fraction of the varying speckle pattern created by the scattered light from the ground glass disk using a $35 \mathrm{~mm}$ lens and a single mode fiber placed $20 \mathrm{~cm}$ away from the ground glass plate. The fiber can be connected to a Hanbury Brown-Twiss setup (temporal resolution: $500 \mathrm{ps)}$ or the cryostat in order to determine the second-order correlation function of the excitation light beam or excite the sample, respectively. For sample excitation, the light is focused onto a single micropillar using a microscope objective with a numerical aperture of 0.42 , which was also used for collecting the emission from the sample. The experimental procedure goes as follows. We use our Martienssen lamp to measure an input-output curve and the second-order correlation function of the emission from the micropillar laser. We then stop the rotation of the ground glass disk and use the light scattered from the stationary disk to measure another input-output curve of the same micropillar under coherent excitation, but otherwise identical excitation conditions. The second-order correlation functions are corrected for the background count rate and the results are compared. The coherence properties of our pseudothermal light source as determined by the rotation velocity, grit polish, and spot position used in this experiment are summarized in Fig. 1. The source shows almost perfect thermal statistics with $g^{(2)}(0)=$ $1.97 \pm 0.02$. The coherence time amounts to $13.7 \mu \mathrm{s}$. The integration times in our experiments are at least 4 orders of magnitude longer, so that the photon number distribution is sampled uniformly. 


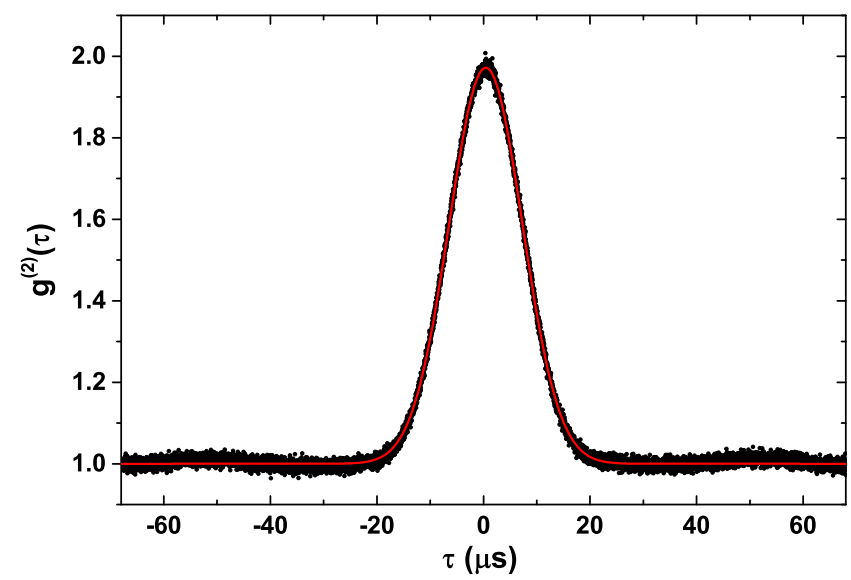

FIG. 1 (color online). Second-order correlation function of the pseudothermal light beam used for excitation, indicating almost perfect thermal photon number statistics. The red line is a Gaussian fit to the data.

A comparison of the $\mathrm{I} / \mathrm{O}$ curves for coherent and thermal excitation is shown in Fig. 2. For thermal excitation, the nonlinear section of the I/O curve begins significantly earlier. Compared to the onset of the threshold region for coherent excitation, which is found at a photon number of $\approx 6 \times 10^{6}$, the threshold is reduced by a factor of about 3 for thermal excitation. This deviation can readily be explained by the large variance of the thermal photon number distribution. Because of the large spread in instantaneous photon numbers, responses within the nonlinear threshold region already gain significant weight in the total response function at much lower mean excitation photon numbers compared to the case of coherent excitation. The secondorder correlation function of the micropillar emission is shown in Fig. 3. Far below and far above the threshold region, the emission photon number statistics reflect the pseudothermal character of the excitation beam, resulting in $g^{(2)}(0)=2$. Within the nonlinear region, a pronounced increase in photon number noise is evident. Values as large as $g^{(2)}(0)=15$ are reached. This result can be understood intuitively: While the photon number distribution is narrow for coherent excitation, the broad thermal input photon number distribution samples a broad range of responses. Because of the presence of the nonlinearity, in the latter case the amplification ratio will depend on the instantaneous input photon number as large photon numbers will be subject to much stronger amplification than low photon numbers, thus effectively increasing the variance of the photon number distribution. In order to test whether the different results can be traced back solely to the different excitation photon number statistics, we also calculate the expected response of the system to thermal excitation from the response to coherent excitation. To this end, we interpolate the coherent $\mathrm{I} / \mathrm{O}$ curve such that we get 8100 equidistant points, each corresponding to an incremental step of $10^{4}$ photons per pulse in the excitation beam. In the

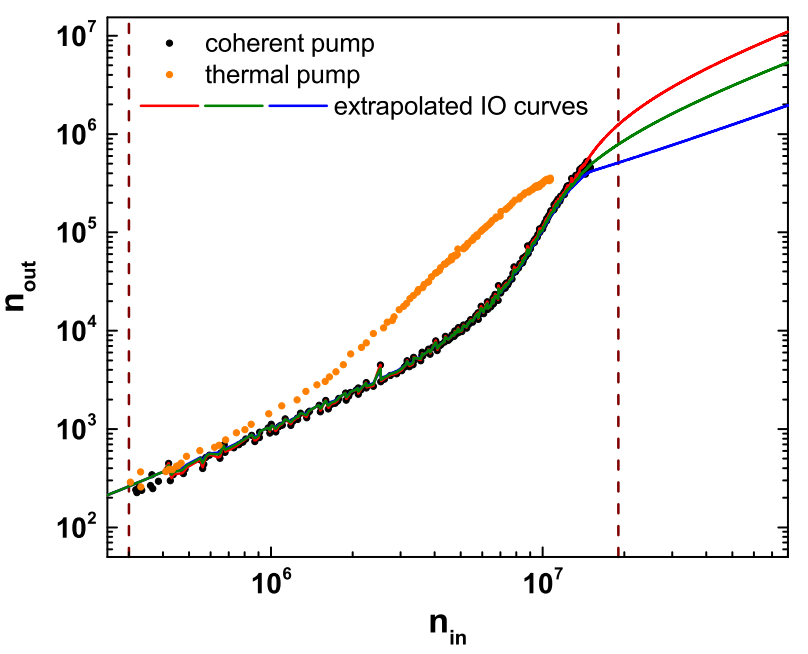

FIG. 2 (color online). Experimentally determined I/O curves for coherent (black dots) and thermal (orange dots) excitation. Solid lines represent three possible extrapolations beyond the range of available excitation powers. Dashed lines indicate the region of excitation powers investigated further in Fig. 3.

next step, we determine the expected response for thermal excitation by projecting the measured $\mathrm{I} / \mathrm{O}$ curve for coherent excitation onto a thermal excitation photon number distribution. To this end, we calculate a weighted superposition of the I/O curve values for coherent excitation using the weight factors of a thermal distribution as given by Eq. (8).

In the case of pseudothermal excitation, the instantaneous photon number may be higher than the mean photon number we can achieve both for thermal or coherent excitation. In order to include the response for large photon numbers into our calculation, we assumed three possible continuations of the I/O curve beyond the measured regime. Those curves differ by the amount of saturation taking place at the upper end of the I/O-curve threshold. However, the probability for the occurrence of these very large instantaneous intensities is very low and becomes completely negligible at photon numbers of about $1.9 \times 10^{7}$, so we introduced a cutoff there. Finally, we compare the measured and the calculated response for thermal excitation. As shown in Fig. 3, the intermediate saturation level reproduces the thermal I/O curve well, while the other choices result in deviations from the measured I/O curve close to the upper end of the nonlinear region. With respect to the second-order correlation function of the emission from the micropillar lasers, all three extrapolated I/O curves predict a significant overshoot in $g^{(2)}(0)$ close to the onset of the threshold. This strong increase in photon number noise is indeed seen in the experiment at the correct position, but the calculated responses tend to slightly overestimate $g^{(2)}(0)$. The best agreement is found for the strongest amount of saturation assumed. However, we must stress that we neglected any influence of the microscopic processes taking place in the 


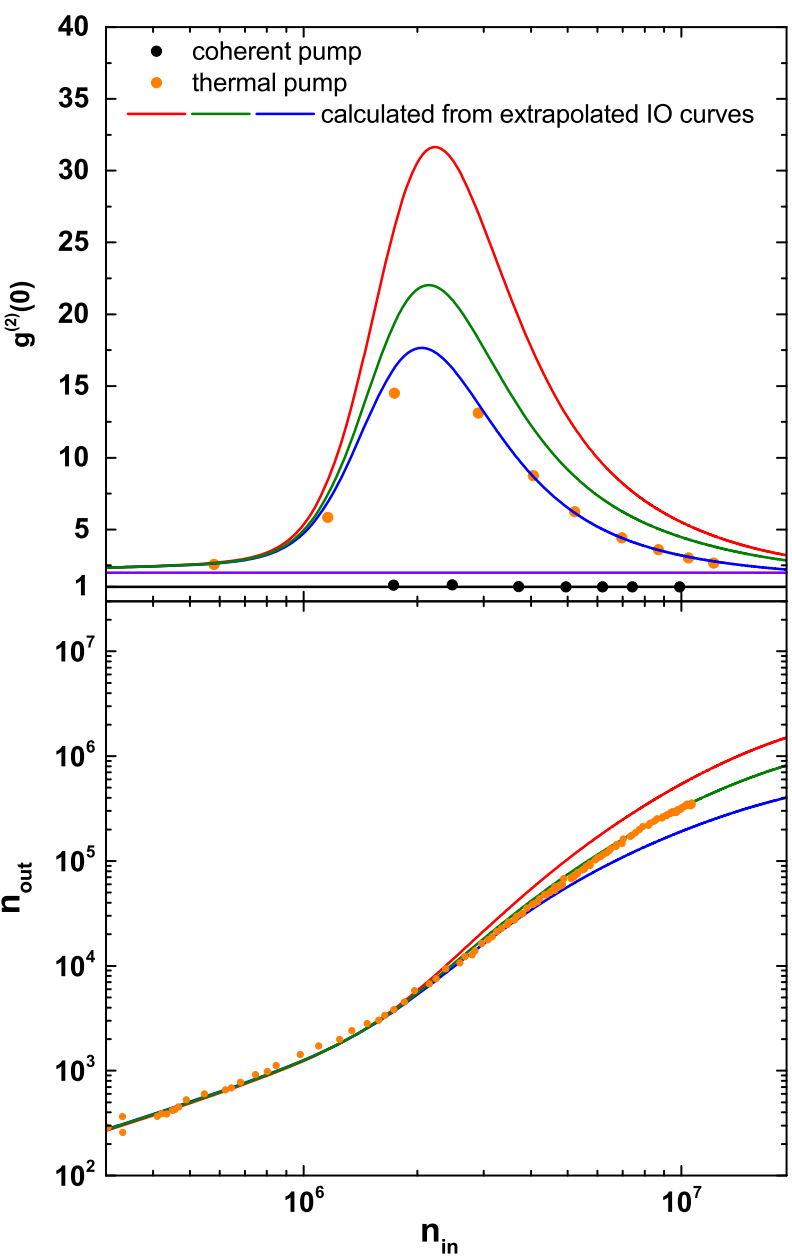

FIG. 3 (color online). Upper panel: Measured $g^{(2)}(0)$ for thermal excitation (orange dots) compared to the calculated $g^{(2)}(0)$ based on the extrapolated coherent I/O curves shown in Fig. 2 projected on a thermal excitation photon number distribution (solid lines). Black dots represent the corresponding measured values for coherent excitation. Because of the temporal resolution of the photodiodes, only negligible amounts of photon bunching show up in these measurements. The black and violet lines represent the coherent and thermal cases of $g^{(2)}(0)=1$ and $g^{(2)}(0)=2$, respectively. Lower panel: Measured I/O curve for thermal excitation (orange dots) compared to the calculated $\mathrm{I} / \mathrm{O}$ curves based on the same coherent $\mathrm{I} / \mathrm{O}$ curves as above (solid lines).

laser operation. We deliberately used photodiodes with a temporal resolution that is not sufficient to measure any short-time effects on the photon statistics of the emitted light, such as photon bunching below threshold [3,23] or relaxation oscillations of the cavity light field [24]. For coherent excitation the largest amount of photon bunching seen in the threshold region amounts to a value of only $g^{(2)}(0)=1.15$. Therefore, only the fundamental differences between coherent and thermal excitation are expected to show up in the experimental results. As the coherence time of the emission increases in the same range of excitation powers where the effect of photon bunching diminishes [25], some small deviations from the predicted behavior are expected. However, a microscopic description is far beyond the scope of this work and it is far more likely that these small deviations arise due to the pseudothermal light source showing an almost, but not absolutely perfect thermal photon number distribution. Our results clearly demonstrate that the output photon number statistics can be explained well just by taking the shape of the I/O curve and the excitation photon number statistics into account.

As a central result, we demonstrated that the input-output curve, which is a standard figure of merit in laser technology, is not necessarily sufficient to capture the key properties of a laser. It is common practice to derive quantities like the $\beta$ factor directly from the I/O curve [26], and our results suggest that the statistics of the pump source need to be taken into account when doing so as even slight imperfections of the excitation laser or current may cause distortions. Photon-statistics excitation spectroscopy allows for a more thorough description of the system response, appears as a discrete variable alternative to quantum process tomography [27], and can be applied to arbitrary systems. Besides the obvious advantages of a full characterization of the response of a system, photonstatistics excitation spectroscopy allows for more complex spectroscopic experiments and opens opportunities for applications. For example, recently, it has been demonstrated that noisy light fields are actually beneficial for two-photon excited fluorescence [28] and the noise level presented here clearly exceeds earlier results [29,30]. Systematic variations of the coherence time of the excitation light field will allow us to study dynamical phenomena like relaxation oscillations. Combining thermal excitation with compressed sensing [31] opens up the opportunity to measure full input-output curves with few measurements. Finally, while coherent excitation of semiconductors creates an optical polarization that is partially converted into optically active excitons, incoherent thermal excitation directly will create only optically active excitons in a degenerate exciton state [15], which is of huge interest in semiconductor physics.

The Dortmund group acknowledges support by the DFG via TRR 142 and research Grant DFG 1549/18-1. The group at Würzburg University acknowledges support by the State of Bavaria.

* Corresponding author. marc.assmann@tu-dortmund.de

[1] P. Michler, A. Imamoğlu, M. D. Mason, P. J. Carson, G. F. Strouse, and S. K. Buratto, Nature (London) 406, 968 (2000).

[2] J. Kasprzak, M. Richard, A. Baas, B. Deveaud, R. André, J.-P. Poizat, and L. S. Dang, Phys. Rev. Lett. 100, 067402 (2008).

[3] M. Aßmann, F. Veit, M. Bayer, M. van der Poel, and J. M. Hvam, Science 325, 297 (2009). 
[4] F. Boitier, A. Godard, E. Rosencher, and C. Fabre, Nat. Phys. 5, 267 (2009).

[5] M. J. Stevens, B. Baek, E. A. Dauler, A. J. Kerman, R. J. Molnar, S. A. Hamilton, K. K. Berggren, R. P. Mirin, and S. W. Nam, Opt. Express 18, 1430 (2010).

[6] E. A. Goldschmidt, F. Piacentini, I. R. Berchera, S. V. Polyakov, S. Peters, S. Kück, G. Brida, I. P. Degiovanni, A. Migdall, and M. Genovese, Phys. Rev. A 88, 013822 (2013).

[7] J. F. Dynes, Z. L. Yuan, A. W. Sharpe, O. Thomas, and A. J. Shields, Opt. Express 19, 13268 (2011).

[8] M. Aßmann, F. Veit, J.-S. Tempel, T. Berstermann, H. Stolz, M. van der Poel, J. M. Hvam, and M. Bayer, Opt. Express 18, 20229 (2010).

[9] N. Takemura, J. Omachi, and M. Kuwata-Gonokami, Phys. Rev. A 85, 053811 (2012).

[10] A. Hayat, A. Nevet, and M. Orenstein, Opt. Lett. 35, 793 (2010).

[11] A. Lebreton, I. Abram, R. Braive, I. Sagnes, I. Robert-Philip, and A. Beveratos, Phys. Rev. Lett. 110, 163603 (2013).

[12] E. del Valle, A. Gonzalez-Tudela, F. P. Laussy, C. Tejedor, and M. J. Hartmann, Phys. Rev. Lett. 109, 183601 (2012).

[13] A. Carmele, A. Knorr, and M. Richter, Phys. Rev. B 79, 035316 (2009).

[14] M. Aßmann and M. Bayer, Phys. Rev. A 84, 053806 (2011).

[15] M. Kira and S. W. Koch, Phys. Rev. A 73, 013813 (2006).

[16] C. Sanchez Munoz, E. del Valle, A. Gonzalez-Tudela, K. Müller, S. Lichtmannecker, M. Kaniber, C. Tejedor, J. Finley, and F. Laussy, Nat. Photonics 8, 550 (2014).

[17] A. Almand-Hunter, H. Li, S. Cundiff, M. Mootz, M. Kira, and S. Koch, Nature (London) 506, 471 (2014).
[18] C. Berger, U. Huttner, M. Mootz, M. Kira, S. W. Koch, J.-S. Tempel, M. Aßmann, M. Bayer, A. M. Mintairov, and J. L. Merz, Phys. Rev. Lett. 113, 093902 (2014).

[19] L. Mandel and E. Wolf, Optical Coherence and Quantum Optics (Cambridge University Press, Cambridge, England, 1995).

[20] G. Galeazzi, A. Lombardi, G. Ruoso, C. Braggio, G. Carugno, F. Della Valle, D. Zanello, and V. V. Dodonov, Phys. Rev. A 88, 053806 (2013).

[21] W. Martienssen and E. Spiller, Am. J. Phys. 32, 919 (1964).

[22] M. Aßmann and M. Bayer, Opt. Lett. 37, 2811 (2012).

[23] S. M. Ulrich, C. Gies, S. Ates, J. Wiersig, S. Reitzenstein, C. Hofmann, A. Löffler, A. Forchel, F. Jahnke, and P. Michler, Phys. Rev. Lett. 98, 043906 (2007).

[24] J. Wiersig, C. Gies, F. Jahnke, M. Aßmann, T. Berstermann, M. Bayer, C. Kistner, S. Reitzenstein, C. Schneider, S. Höfling, A. Forchel, C. Kruse, J. Kalden, and D. Hommel, Nature (London) 460, 245 (2009).

[25] J.-S. Tempel, I. A. Akimov, M. Aßmann, C. Schneider, S. Höfling, C. Kistner, S. Reitzenstein, L. Worschech, A. Forchel, and M. Bayer, J. Opt. Soc. Am. B 28, 1404 (2011).

[26] C. Gies, J. Wiersig, M. Lorke, and F. Jahnke, Phys. Rev. A 75, 013803 (2007).

[27] M. Lobino, D. Korystov, C. Kupchak, E. Figueroa, B. Sanders, and A. Lvovsky, Science 322, 563 (2008).

[28] A. Jechow, M. Seefeldt, H. Kurzke, A. Heuer, and R. Menzel, Nat. Photonics 7, 973 (2013).

[29] F. Albert, C. Hopfmann, S. Reitzenstein, C. Schneider, S. Höfling, L. Worschech, M. Kamp, W. Kinzel, A. Forchel, and I. Kanter, Nat. Commun. 2, 366 (2011).

[30] F. Boitier, A. Godard, N. Dubreuil, P. Delaye, C. Fabre, and E. Rosencher, Nat. Commun. 2, 425 (2011).

[31] D. Donoho, IEEE Trans. Inf. Theory 52, 1289 (2006). 